\title{
EXISTENCE OF A PERIODIC SOLUTION FOR CONTINUOUS AND DISCRETE PERIODIC SECOND-ORDER EQUATIONS WITH VARIABLE POTENTIALS
}

\author{
DOUGLAS R. ANDERSON AND RICHARD I. AVERY
}

\begin{abstract}
Green's functions for new second-order periodic differential and difference equations with variable potentials are found, then used as kernels in integral operators to guarantee the existence of a positive periodic solution to continuous and discrete second-order periodic boundary value problems with periodic coefficient functions. A new version of the Leggett-Williams fixed point theorem is employed.
\end{abstract}

1. Introduction to the Continuous Periodic Problem

Consider the new second-order differential equation with variable potentials given by

$$
L y(t)=-\left(p y^{\prime}\right)^{\prime}(t)-r(t) p(t) y^{\prime}(t)+q(t) y(t)=f(t, y(t)), \quad t \in[0, \infty),
$$

with the periodic boundary conditions

$$
y(t)=y(t+\omega), \quad y^{\prime}(t)=y^{\prime}(t+\omega), \quad t \in[0, \infty),
$$

where $\omega>0$ is the period, and we assume the hypotheses

$\left(H_{1}\right) p, q, r \in \mathrm{C}(\mathbb{R})$ with $p(t), q(t)>0$ and $r(t) \geq 0$ for all $t \in[0, \infty)$;

$\left(H_{2}\right) p(t)=p(t+\omega), q(t)=q(t+\omega), r(t)=r(t+\omega)$, for all $t \in[0, \infty)$;

$\left(H_{3}\right) f:[0, \infty) \times[0, \infty) \rightarrow[0, \infty)$ is continuous with $f(t, z)=f(t+\omega, z)$.

There are several recent results on second-order periodic problems. Liu, Ge, and Gui [13] (see also [4]) consider problem (1.1), (1.2) with $r(t) \equiv 0$. Graef, Kong, Wang [7] give an extensive analysis for a constant coefficient case,

$$
y^{\prime \prime}(t)-\rho^{2} y(t)+\lambda g(t) f(y)=0, \quad t \in[0,2 \pi]
$$

with periodic boundary conditions

$$
y(0)=y(2 \pi), \quad y^{\prime}(0)=y^{\prime}(2 \pi) .
$$

2000 Mathematics Subject Classification. 34B15, 39A10.

Key words and phrases. boundary value problems, Riccati equation, fixed points, difference equations, differential equations. 
Thus (1.1), (1.2) extend both of these papers directly. Torres [14] and Chu, Torres, and Zhang [6] study the existence of periodic solutions to

$$
\pm y^{\prime \prime}(t)+a(t) y(t)=f(t, y(t))+e(t)
$$

by making use of assumptions on the (then unknown) Green's function for

$$
y^{\prime \prime}(t)+a(t) y(t)=0, \quad y(0)=y(T), \quad y^{\prime}(0)=y^{\prime}(T)
$$

see the explicit formula for the Green's function in (2.6) below. Moreover, unlike [6, 7, 13, 14] we will also deal with the discrete analogue of these results below in Section 4. First, in Section 2 we introduce our main insight, the connection between solutions of (1.1), (1.2) and a related Ricccati equation; in Section 3 we discuss and employ a recent extension of the Leggett-Williams fixed point theorem which requires neither of the functional boundaries to be invariant [5].

\section{Preliminary Results}

In this section we will prove the existence of a periodic solution to a Riccati equation associated with $L y(t)=0$, and use this Riccati function in the choice of an exponential function that will allow us to factor and integrate the operator equation

$$
\left(p y^{\prime}\right)^{\prime}(t)+p(t) r(t) y^{\prime}(t)-q(t) y(t)=0
$$

This in turn will lead to a nice expression for the corresponding Green's function for $L y=0$ with boundary conditions (1.2) in such a way that we can find bounds on it for later use.

Remark 2.1. Assume that $\left(H_{1}\right)$ and $\left(H_{2}\right)$ hold. We define

$$
\begin{aligned}
p_{*} & =\min _{t \in[0, \omega]} p(t), \quad q_{*}=\min _{t \in[0, \omega]} q(t), \quad r_{*}=\min _{t \in[0, \omega]} r(t), \\
p^{*} & =\max _{t \in[0, \omega]} p(t), \quad q^{*}=\max _{t \in[0, \omega]} q(t), \quad r^{*}=\max _{t \in[0, \omega]} r(t), \\
\Gamma_{*} & =\frac{-p_{*} r^{*}+\sqrt{\left(p_{*} r^{*}\right)^{2}+4 p_{*} q_{*}}}{2}, \quad \Gamma^{*}=\frac{-p^{*} r_{*}+\sqrt{\left(p^{*} r_{*}\right)^{2}+4 p^{*} q^{*}}}{2} .
\end{aligned}
$$

Then we have the following Riccati result.

Theorem 2.2. Assume $\left(H_{1}\right),\left(H_{2}\right)$. If the potentials $p, q, r$ are such that $\Gamma_{*} \leq \Gamma^{*}$, then the $\omega$-periodic Riccati differential equation

$$
z^{\prime}(t)=q(t)-r(t) z(t)-\frac{z^{2}(t)}{p(t)}, \quad t \in[0, \infty)
$$

has an attracting positive $\omega$-periodic solution $\bar{z}$ such that $\bar{z}(t) \in\left[\Gamma_{*}, \Gamma^{*}\right]$ for all $t \in[0, \infty)$. 
Proof. Clearly $0<\Gamma_{*} \leq \Gamma^{*}$. For $z_{0} \in \mathbb{R}$, let $z\left(t ; 0, z_{0}\right)$ be the unique solution of $(2.2)$ through the point $\left(0, z_{0}\right)$. If $z\left(t ; 0, z_{0}\right)=z(t) \in\left(0, \Gamma_{*}\right]$ for some $t \in[0, \omega]$, then

$$
z^{\prime}(t)=q(t)-r(t) z(t)-\frac{z^{2}(t)}{p(t)} \geq q_{*}-r^{*} \Gamma_{*}-\frac{\Gamma_{*}^{2}}{p_{*}}=0
$$

by the definition of $\Gamma_{*}$, and $z$ is nondecreasing at that point. In the same way, if $z\left(t ; 0, z_{0}\right)=z(t) \in\left[\Gamma^{*}, \infty\right)$ for some $t \in[0, \omega]$, then

$$
z^{\prime}(t)=q(t)-r(t) z(t)-\frac{z^{2}(t)}{p(t)} \leq q^{*}-r_{*} \Gamma^{*}-\frac{\Gamma^{* 2}}{p^{*}}=0
$$

by the definition of $\Gamma^{*}$, and $z$ is nonincreasing at that point. Therefore by the direction of the vector field, if $z_{0} \in\left[\Gamma_{*}, \Gamma^{*}\right]$, then $z(t) \in\left[\Gamma_{*}, \Gamma^{*}\right]$ for all $t \in \mathbb{R}$.

Note that in particular, $z\left(\omega ; 0, z_{0}\right) \in\left[\Gamma_{*}, \Gamma^{*}\right]$. This motivates us to define the operator $\Psi:\left[\Gamma_{*}, \Gamma^{*}\right] \rightarrow\left[\Gamma_{*}, \Gamma^{*}\right]$ via $\Psi\left(z_{0}\right)=z\left(\omega ; 0, z_{0}\right)$ for each $z_{0} \in\left[\Gamma_{*}, \Gamma^{*}\right]$. Since $\Psi$ is a continuous bijection on a compact interval, it has a fixed point $z_{0}^{*}$. Since $p, q$, and $r$ are $\omega$-periodic functions, the unique solution $\bar{z}:=\bar{z}\left(\cdot ; 0, z_{0}^{*}\right)$ of the Riccati equation $(2.2)$ through $\left(0, z_{0}^{*}\right)$ is positive and $\omega$-periodic. To show that $\bar{z}$ is the unique, attracting, $\omega$-periodic solution, let $z$ be any other positive solution of $(2.2)$ with initial condition in $\left[\Gamma_{*}, \Gamma^{*}\right]$, and let $u:=\bar{z}-z$. Then

$$
u^{\prime}=\phi u, \quad u=\bar{z}-z,
$$

where

$$
\phi:=-\left(r+\frac{\bar{z}+z}{p}\right) \in\left[-\left(r^{*}+\frac{2 \Gamma^{*}}{p_{*}}\right),-\left(r_{*}+\frac{2 \Gamma_{*}}{p^{*}}\right)\right]
$$

is negative and bounded away from zero. In particular, $\phi(t)<0$ and $u$ and $u^{\prime}$ are of opposite sign for all $t \in \mathbb{R}$. Therefore

$$
u(t)=u(0) e^{\int_{0}^{t} \phi(s) d s} \quad \text { with } \quad u(0)=\bar{z}(0)-z(0) .
$$

It follows that $u(t)=\bar{z}(t)-z(t)=u(0) e^{\int_{0}^{t} \phi(s) d s}$ is of constant sign on $\mathbb{R}$, and since $u$ and $u^{\prime}$ are of opposite sign, $\lim _{t \rightarrow \infty} u(t)$ exists in $\mathbb{R}$, and

$$
\lim _{t \rightarrow \infty} u^{\prime}(t)=\lim _{t \rightarrow \infty} \phi(t) u(t)=0 .
$$

As $\phi(t)$ does not go to 0 by the positivity of $z, \bar{z}, p$, and $r$ and the periodicity of $\bar{z}, p$, and $r$, we have $z(t) \rightarrow \bar{z}(t)$. Consequently, $\bar{z}$ is the unique positive, $\omega$-periodic, attracting (asymptotically stable) solution to (2.2).

Remark 2.3. If the potentials $p, q, r$ are all constant on $\mathbb{R}$ with $p \equiv 1$, then the Riccati equation (2.2) has a constant attracting solution given by

$$
\bar{z}=\Gamma_{*}=\Gamma^{*}=\frac{-r+\sqrt{r^{2}+4 q}}{2} .
$$


Theorem 2.4. Assume $\left(H_{1}\right),\left(H_{2}\right)$. Further assume that the potentials $p, q, r$ are such that $\Gamma_{*} \leq \Gamma^{*}$, where $\Gamma_{*}$ and $\Gamma^{*}$ are given in Theorem 2.2. Let $h: \mathbb{R} \rightarrow[0, \infty)$ be an $\omega$-periodic continuous function, and let $z$ be the attracting positive $\omega$-periodic solution of (2.2). Then the periodic boundary value problem

$$
\left(p y^{\prime}\right)^{\prime}(t)+r(t) p(t) y^{\prime}(t)-q(t) y(t)+h(t)=0, \quad t \in[0, \infty)
$$

with periodic boundary conditions

$$
y(t)=y(t+\omega), \quad y^{\prime}(t)=y^{\prime}(t+\omega), \quad t \in[0, \infty),
$$

has periodic solution

$$
y(t)=\int_{t}^{t+\omega} G(t, s) h(s) d s
$$

for the Green's function $G(t, s)$ given by

$$
G(t, s):=\int_{t}^{s} \frac{\exp \left[\int_{\eta}^{t+\omega} \frac{z(j)}{p(j)} d j+\int_{\eta}^{s} \xi(j) d j\right]}{k p(\eta)} d \eta+\int_{s}^{t+\omega} \frac{\exp \left[\int_{\eta}^{t+\omega} \frac{z(j)}{p(j)} d j+\int_{\eta}^{s+\omega} \xi(j) d j\right]}{k p(\eta)} d \eta,
$$

where $t \in[0, \infty), s \in[t, t+\omega]$, the positive continuous function $\xi$ is given by

$$
\xi(t):=\frac{z(t)}{p(t)}+r(t)>0, \quad t \in \mathbb{R}
$$

and the constant $k$ is given by

$$
k:=\left(\exp \left[\int_{0}^{\omega} \frac{z(j)}{p(j)} d j\right]-1\right)\left(\exp \left[\int_{0}^{\omega}\left(\frac{z(j)}{p(j)}+r(j)\right) d j\right]-1\right)>0 .
$$

Proof. Let $z$ be the attracting positive $\omega$-periodic solution to the Riccati equation found in Theorem 2.2. We use an integrating factor of the form

$$
E(t) \equiv E(t, 0 ; \xi):=\exp \left[\int_{0}^{t} \xi(j) d j\right]
$$

for $\xi$ given in (2.7), and consider the following factored differential equation, where for brevity the independent variable $t$ and the initial time 0 have been suppressed:

$$
\left(p y^{\prime} E\right)^{\prime}-(z y E)^{\prime}=-h E
$$

Expanding this out and simplifying via (2.7) we obtain

$$
\begin{aligned}
\left(p y^{\prime}\right)^{\prime}+p y^{\prime} \xi-z y^{\prime}-z^{\prime} y-z y \xi & =-h \\
\left(p y^{\prime}\right)^{\prime}+r p y^{\prime}-\left(z^{\prime}+\frac{z^{2}}{p}+z r\right) y & =-h \\
\left(p y^{\prime}\right)^{\prime}+r p y^{\prime}-q y & =-h,
\end{aligned}
$$


which is (2.4) since $z$ is a solution of (2.2). Integrate (2.9) from $t$ to $t+\omega$ and use the periodicity of $p, y, y^{\prime}$, and $z$ to get

$$
\left[p(t) y^{\prime}(t)-z(t) y(t)\right][E(t+\omega)-E(t)]=-\int_{t}^{t+\omega} h(v) E(v) d v .
$$

Note that $E(t+\omega)-E(t)=E(t)(E(\omega)-1)$. This implies that

$$
y^{\prime}(t)=\frac{z(t)}{p(t)} y(t)+\frac{-1}{p(t)[E(\omega)-1]} \int_{t}^{t+\omega} h(v) \frac{E(v)}{E(t)} d v .
$$

For the next few steps, temporarily set

$$
\zeta(\eta):=\frac{-1}{p(\eta)[E(\omega)-1]} \int_{\eta}^{\eta+\omega} h(v) \frac{E(v)}{E(\eta)} d v .
$$

By the variation of constants formula (2.10) has the solution

$$
y(t+\omega)=\exp \left[\int_{t}^{t+\omega} \frac{z(j)}{p(j)} d j\right] y(t)+\int_{t}^{t+\omega} \exp \left[\int_{\eta}^{t+\omega} \frac{z(j)}{p(j)} d j\right] \zeta(\eta) d \eta .
$$

Since

we obtain

$$
y(t+\omega)=y(t) \quad \text { and } \quad \exp \left[\int_{t}^{t+\omega} \frac{z(j)}{p(j)} d j\right]=\exp \left[\int_{a}^{\omega} \frac{z(j)}{p(j)} d j\right]
$$

$$
y(t)=\frac{1}{1-\exp \left[\int_{0}^{\omega} \frac{z(j)}{p(j)} d j\right]} \int_{t}^{t+\omega} \exp \left[\int_{\eta}^{t+\omega} \frac{z(j)}{p(j)} d j\right] \zeta(\eta) d \eta,
$$

which can be rewritten as

$$
y(t)=\frac{-1}{1-\exp \left[-\int_{0}^{\omega} \frac{z(j)}{p(j)} d j\right]} \int_{t}^{t+\omega} \exp \left[\int_{\eta}^{t} \frac{z(j)}{p(j)} d j\right] \zeta(\eta) d \eta .
$$

Thus a solution of (2.4) with periodic boundary conditions (1.2) is given by

$$
y(t)=\int_{t}^{t+\omega} \int_{\eta}^{\eta+\omega} \frac{\exp \left[\int_{\eta}^{t} \frac{z(j)}{p(j)} d j\right] \exp \left[\int_{\eta}^{v} \xi(j) d j\right] h(v)}{p(\eta)\left(1-\exp \left[-\int_{0}^{\omega} \frac{z(j)}{p(j)} d j\right]\right)\left(\exp \left[\int_{0}^{\omega} \xi(j) d j\right]-1\right)} d v d \eta,
$$

where $\xi$ is given in (2.7). Interchange the order of integration to obtain

$$
\begin{aligned}
y(t)= & \int_{t}^{t+\omega}\left(\int_{t}^{v} \frac{\exp \left[\int_{\eta}^{t} \frac{z(j)}{p(j)} d j\right] \exp \left[\int_{\eta}^{v} \xi(j) d j\right] h(v)}{p(\eta)\left(1-\exp \left[-\int_{0}^{\omega} \frac{z(j)}{p(j)} d j\right]\right)\left(\exp \left[\int_{0}^{\omega} \xi(j) d j\right]-1\right)} d \eta\right) h(v) d v \\
& +\int_{t+\omega}^{t+2 \omega}\left(\int_{v-\omega}^{t+\omega} \frac{\exp \left[\int_{\eta}^{t} \frac{z(j)}{p(j)} d j\right] \exp \left[\int_{\eta}^{v} \xi(j) d j\right] h(v)\left(1-\exp \left[-\int_{0}^{\omega} \frac{z(j)}{p(j)} d j\right]\right)\left(\exp \left[\int_{0}^{\omega} \xi(j) d j\right]-1\right)}{p(\eta)} d \eta\right) h(v) d v
\end{aligned}
$$

Employing the substitutions $s=v$ and $s=v-\omega$, we arrive at

$$
y(t)=\int_{t}^{t+\omega} G(t, s) h(s) d s,
$$

where $G(t, s)$ is given by (2.6) for $t \in[0, \infty)$ and $s \in[t, t+\omega]$. 
Corollary 2.5. Assume the hypotheses of Theorem 2.4. Then the periodic self-adjoint boundary value problem

$$
\left(p y^{\prime}\right)^{\prime}(t)-q(t) y(t)+h(t)=0, \quad t \in[0, \infty)
$$

with periodic boundary conditions

$$
y(t)=y(t+\omega), \quad y^{\prime}(t)=y^{\prime}(t+\omega), \quad t \in[0, \infty)
$$

has periodic solution

$$
y(t)=\int_{t}^{t+\omega} G(t, s) h(s) d s
$$

for the Green's function $G(t, s)$ given by

$$
G(t, s):=\int_{t}^{s} \frac{\exp \left[\int_{\eta}^{t+\omega} \frac{z(j)}{p(j)} d j+\int_{\eta}^{s} \frac{z(j)}{p(j)} d j\right]}{p(\eta)\left(\exp \left[\int_{0}^{\omega} \frac{z(j)}{p(j)} d j\right]-1\right)^{2}} d \eta+\int_{s}^{t+\omega} \frac{\exp \left[\int_{\eta}^{t+\omega} \frac{z(j)}{p(j)} d j+\int_{\eta}^{s+\omega} \frac{z(j)}{p(j)} d j\right]}{p(\eta)\left(\exp \left[\int_{0}^{\omega} \frac{z(j)}{p(j)} d j\right]-1\right)^{2}} d \eta
$$

where $t \in[0, \infty)$ and $s \in[t, t+\omega]$.

Corollary 2.6. Assume the hypotheses of Theorem 2.4. Further assume that the potentials $p, q, r$ are all constant on $\mathbb{R}$ with $p \equiv 1$. Then the constant coefficient boundary value problem

$$
y^{\prime \prime}(t)+r y^{\prime}(t)-q y(t)+h(t)=0, \quad t \in[0, \infty)
$$

with periodic boundary conditions

$$
y(t)=y(t+\omega), \quad y^{\prime}(t)=y^{\prime}(t+\omega), \quad t \in[0, \infty),
$$

has periodic solution

$$
y(t)=\int_{t}^{t+\omega} G(t, s) h(s) d s
$$

for the Green's function $G(t, s)$ given by

$$
G(t, s):=\int_{t}^{s} \frac{e^{\bar{z}(t+s+\omega-2 \eta)+r(s-\eta)}}{\left(e^{\bar{z} \omega}-1\right)\left(e^{(\bar{z}+r) \omega}-1\right)} d \eta+\int_{s}^{t+\omega} \frac{e^{\bar{z}(t+\omega-\eta)+(\bar{z}+r)(s+\omega-\eta)}}{\left(e^{\bar{z} \omega}-1\right)\left(e^{(\bar{z}+r) \omega}-1\right)} d \eta,
$$

where $t \in[0, \infty), s \in[t, t+\omega]$ and $\bar{z}$ is given in $(2.3)$.

Lemma 2.7. Assume $\left(H_{1}\right)$ and $\left(H_{2}\right)$. Let $h: \mathbb{R} \rightarrow[0, \infty)$ be continuous, and let $z, y$ be the solutions of (2.2), (2.4) respectively. Then

$$
y(t) \geq \ell\|y\| \quad \text { for } \quad t \in[0, \omega]
$$

where the constant $\ell$ is given by

$$
\ell:=\frac{1}{\exp \left[\int_{0}^{\omega} \xi(j) d j\right]\left(1+\exp \left[\int_{0}^{\omega} \xi(j) d j\right]\right)}
$$


Proof. For the kernel $G$ given in (2.6), we have for $t \leq s \leq t+\omega$ that

$$
\begin{aligned}
G(t, s) & =\int_{t}^{s} \frac{\exp \left[\int_{\eta}^{t+\omega} \frac{z(j)}{p(j)} d j+\int_{\eta}^{s} \xi(j) d j\right]}{k p(\eta)} d \eta+\int_{s}^{t+\omega} \frac{\exp \left[\int_{\eta}^{t+\omega} \frac{z(j)}{p(j)} d j+\int_{\eta}^{s+\omega} \xi(j) d j\right]}{k p(\eta)} d \eta \\
& \leq \int_{t}^{t+\omega} \frac{\exp \left[\int_{\eta}^{t+\omega} \frac{z(j)}{p(j)} d j\right]}{k p(\eta)}\left(\exp \left[\int_{\eta}^{s} \xi(j) d j\right]+\exp \left[\int_{\eta}^{s+\omega} \xi(j) d j\right]\right) d \eta \\
& \leq \exp \left[\int_{t}^{s} \xi(j) d j\right]\left(1+\exp \left[\int_{s}^{s+\omega} \xi(j) d j\right]\right) \int_{t}^{t+\omega} \frac{\exp \left[\int_{\eta}^{t+\omega} \frac{z(j)}{p(j)} d j\right]}{k p(\eta)} d \eta \\
& \leq \exp \left[\int_{0}^{\omega} \xi(j) d j\right]\left(1+\exp \left[\int_{0}^{\omega} \xi(j) d j\right]\right) \int_{0}^{\omega} \frac{\exp \left[\int_{\eta}^{\omega} \frac{z(j)}{p(j)} d j\right]}{k p(\eta)} d \eta
\end{aligned}
$$

by the periodicity of the functions involved. Likewise

$$
\begin{aligned}
G(t, s) & =\int_{t}^{s} \frac{\exp \left[\int_{\eta}^{t+\omega} \frac{z(j)}{p(j)} d j+\int_{\eta}^{s} \xi(j) d j\right]}{k p(\eta)} d \eta+\int_{s}^{t+\omega} \frac{\exp \left[\int_{\eta}^{t+\omega} \frac{z(j)}{p(j)} d j+\int_{\eta}^{s+\omega} \xi(j) d j\right]}{k p(\eta)} d \eta \\
& \geq \int_{t}^{t+\omega} \frac{\exp \left[\int_{\eta}^{t+\omega} \frac{z(j)}{p(j)} d j\right]}{k p(\eta)} d \eta=\int_{0}^{\omega} \frac{\exp \left[\int_{\eta}^{\omega} \frac{z(j)}{p(j)} d j\right]}{k p(\eta)} d \eta
\end{aligned}
$$

If we define

$$
g_{*}:=\int_{0}^{\omega} \frac{\exp \left[\int_{\eta}^{\omega} \frac{z(j)}{p(j)} d j\right]}{k p(\eta)} d \eta
$$

and

$$
g^{*}:=\exp \left[\int_{0}^{\omega} \xi(j) d j\right]\left(1+\exp \left[\int_{0}^{\omega} \xi(j) d j\right]\right) \int_{0}^{\omega} \frac{\exp \left[\int_{\eta}^{\omega} \frac{z(j)}{p(j)} d j\right]}{k p(\eta)} d \eta
$$

then

$$
y(t) \geq \frac{g_{*}}{g^{*}}\|y\|=\ell\|y\|, \quad t \in[0, \infty)
$$

This completes the proof.

In the next section we introduce the fixed point theorem that we will employ, namely a recent extension of a Leggett and Williams theorem, for our main existence result.

\section{Existence of a Periodic Solution}

In this section, we establish our main existence result by applying Theorem 3.2 to guarantee the existence of a solution to $(1.1),(1.2)$ in a certain cone. We begin with some necessary definitions. 
Definition 3.1. A map $\alpha$ is said to be a nonnegative continuous concave functional on a cone $P$ of a real Banach space $E$ if

$$
\alpha: P \rightarrow[0, \infty)
$$

is continuous and

$$
\alpha(t x+(1-t) y) \geq t \alpha(x)+(1-t) \alpha(y)
$$

for all $x, y \in P$ and $t \in[0,1]$. Similarly we say the map $\beta$ is a nonnegative continuous convex functional on a cone $P$ of a real Banach space $E$ if

$$
\beta: P \rightarrow[0, \infty)
$$

is continuous and

$$
\beta(t x+(1-t) y) \leq t \beta(x)+(1-t) \beta(y)
$$

for all $x, y \in P$ and $t \in[0,1]$.

Let $\alpha$ and $\psi$ be non-negative continuous concave functionals on $P$ and $\delta$ and $\beta$ be non-negative continuous convex functionals on $P$; then, for non-negative real numbers $a, b, c$ and $d$, we define the following sets:

$$
\begin{aligned}
A & :=A(\alpha, \beta, a, d)=\{y \in P: a \leq \alpha(y) \text { and } \beta(y) \leq d\} \\
B & :=B(\alpha, \delta, \beta, a, b, d)=\{y \in A: \delta(y) \leq b\} \\
C & :=C(\alpha, \psi, \beta, a, c, d)=\{y \in A: c \leq \psi(y)\} .
\end{aligned}
$$

We say that $A$ is a functional wedge with concave functional boundary defined by the concave functional $\alpha$ and convex functional boundary defined by the convex functional $\beta$. We say that an operator $T: A \rightarrow P$ is $i n v a r i a n t$ with respect to the concave functional boundary, if $a \leq \alpha(T y)$ for all $y \in A$, and that $T$ is invariant with respect to the convex functional boundary, if $\beta(T y) \leq d$ for all $y \in A$. Note that $A$ is a convex set. The following theorem is an extension of the original Leggett-Williams fixed point theorem [11].

Theorem 3.2. [Extension of Leggett-Williams] Suppose $P$ is a cone in a real Banach space E, $\alpha$ and $\psi$ are non-negative continuous concave functionals on $P, \delta$ and $\beta$ are non-negative continuous convex functionals on $P$, and for non-negative real numbers $a, b, c$ and $d$ the sets $A, B$ and $C$ are as defined in (3.1), (3.2) and (3.3), respectively. Furthermore, suppose that $A$ is a bounded subset of $P$, that $T: A \rightarrow P$ is completely continuous and that the following conditions hold:

$(A 1)\{y \in A: c<\psi(y)$ and $\delta(y)<b\} \neq \emptyset$ and $\{y \in P: \alpha(y)<a$ and $d<\beta(y)\}=\emptyset$;

(A2) $\alpha(T y) \geq$ a for all $y \in B$;

(A3) $\alpha(T y) \geq a$ for all $y \in A$ with $\delta(T y)>b$; 
(A4) $\beta(T y) \leq d$ for all $y \in C$; and,

(A5) $\beta(T y) \leq d$ for all $y \in A$ with $\psi(T y)<c$.

Then $T$ has a fixed point $y^{*} \in A$.

To be able to apply Theorem 3.2 to the periodic problem (1.1), (1.2), we need the following cone structure. Let $E$ denote the Banach space $\mathrm{C}([0, \infty))$ with the supremum norm

$$
\|y\|=\sup _{t \in[0, \omega]}|y(t)|,
$$

and for $\ell$ given in (2.13) define the cone $P \subset E$ via

$$
P:=\left\{y \in E: y(t) \geq \ell\|y\|, y(t+\omega)=y(t) \text { and } y^{\prime}(t+\omega)=y^{\prime}(t) \text { for all } t \in[0, \infty)\right\} .
$$

Then the fixed points of the integral operator $T: P \rightarrow E$ given by

$$
T y(t):=\int_{t}^{t+\omega} G(t, s) f(s, y(s)) d s
$$

are solutions of (1.1), (1.2) by Theorem 2.4, where $G(t, s)$ is from (2.6). Using (3.5), let the nonnegative continuous concave functional $\psi: P \rightarrow[0, \infty)$ be defined by

$$
\psi(y)=\min _{t \in[0, \omega]} y(t), \quad y \in P ;
$$

note that for $y \in P, 0<\psi(y) \leq\|y\|$ by Lemma 2.7. Furthermore, let the linear functionals (thus both concave and convex) $\alpha, \beta: P \rightarrow[0, \infty)$ be defined by

$$
\beta(y)=\alpha(y)=\frac{1}{\omega} \int_{0}^{\omega} y(t) d t
$$

and let $\delta: P \rightarrow[0, \infty)$ be defined by $\delta(y)=\|y\|$, making $\delta$ a non-negative continuous convex functional.

In the following theorem, we demonstrate how to apply the Extension of the Leggett-Williams Fixed Point Theorem (Theorem 3.2), to prove the existence of at least one positive solution to (1.1), (1.2).

Theorem 3.3. Assume $\left(H_{1}\right)-\left(H_{3}\right)$. For any $d>0$, suppose the following hold:

(a) $f(t, y) \geq m_{l} y+b_{l}$ for $y \in\left[\ell^{3} d, \ell d\right]$, for all $t \in[0, \omega]$,

(b) $f(t, y) \leq m_{l}(\ell d)+b_{l}$ for $y \in\left[\ell^{2} d, d / \ell\right]$, for all $t \in[0, \omega]$,

where for $\ell, g_{*}$ and $g^{*}$ given in (2.13), (2.14) and (2.15), respectively, we have taken

$$
m_{l}=\frac{1}{2 g^{*} \omega}>0 \quad \text { and } \quad b_{l}=\frac{\ell^{2} d\left(1-\frac{1}{2} \ell\right)}{\omega g_{*}}>0 .
$$

Then the operator $T$ has at least one positive solution $y^{*} \in A\left(\alpha, \beta, \ell^{2} d, d\right)$, and thus problem (1.1), (1.2) has at least one positive solution. 
Proof. For any $d>0$ and $\ell$ in (2.13), let $a=c=\ell^{2} d, b=\ell d$. By the properties of $G(t, s)$ given in Theorem 2.4 and Lemma 2.7, we have $T: A(\alpha, \beta, a, d) \rightarrow P$. Using the Arzela-Ascoli Theorem it is a standard exercise to show that $T$ is a completely continuous operator from the properties of $G$ and $f$, and by the definition of $\beta$, we have that $A$ is a bounded subset of the cone $P$. If $y \in P$ and $\beta(y)>d$, then $\alpha(y)=\beta(y)>d>\ell^{2} d=a$. Therefore, $\{y \in P: \alpha(y)<a$ and $d<\beta(y)\}=\emptyset$. Define the constant function $y_{0}:=\frac{1}{2}(c+b)$. Then $\alpha\left(y_{0}\right)=y_{0}=\frac{1}{2}(a+b) \geq a$ and $\beta\left(y_{0}\right)=y_{0}=\frac{1}{2}\left(\ell^{2} d+\ell d\right) \leq d$ since $\ell \in(0,1)$, putting $y_{0} \in A$. Moreover, $\psi\left(y_{0}\right)=y_{0}>c, \delta\left(y_{0}\right)=y_{0}<b$, and thus

$$
\{y \in A: c<\psi(y) \text { and } \delta(y)<b\} \neq \emptyset .
$$

Claim 1: $\alpha(T y) \geq a$ for all $y \in B$.

For any $y \in B$, there is a $t_{0} \in[0, \omega]$ such that $y\left(t_{0}\right)=\alpha(y) \geq a$. It follows that for $y \in B$,

$$
\ell d=b \geq \delta(y)=\|y\| \geq \alpha(y)=y\left(t_{0}\right) \geq \psi(y) \geq \ell\|y\| \geq \ell \alpha(y) \geq \ell a=\ell^{3} d
$$

and therefore $\psi(y) \geq \ell a=\ell^{3} d$. Thus, for all $y \in B$ we have $\ell^{3} d \leq y(t) \leq \ell d$ for all $t \in[0, \infty)$. Consequently by condition (a),

$$
\begin{aligned}
\alpha(T y) & =\frac{1}{\omega} \int_{0}^{\omega} \int_{t}^{t+\omega} G(t, s) f(s, y(s)) d s d t \geq \frac{g_{*}}{\omega} \int_{0}^{\omega} \int_{t}^{t+\omega} f(s, y(s)) d s d t \\
& =\frac{g_{*}}{\omega} \int_{0}^{\omega} \int_{0}^{\omega} f(s, y(s)) d s d t=g_{*} \int_{0}^{\omega} f(s, y(s)) d s \\
& \geq g_{*} \int_{0}^{\omega}\left(m_{l} y(s)+b_{l}\right) d s=g_{*} \omega\left(m_{l} \alpha(y)+b_{l}\right) \\
& \geq g_{*} \omega\left(m_{l} a+b_{l}\right)=a
\end{aligned}
$$

using $a=\ell^{2} d$ and (3.9).

Claim 2: $\alpha(T y) \geq a$, for all $y \in A$ with $\delta(T y)>b$.

Let $y \in A$ with $\delta(T y)>b$. Thus by Lemma 2.7, Ty(s) $\geq \ell b$ for all $s \in[0, \omega]$, so that

$$
\alpha(T y)=\frac{1}{\omega} \int_{0}^{\omega} T y(s) d s \geq \frac{1}{\omega} \int_{0}^{\omega} \ell b d s=\ell b=a .
$$

Claim 3: $\beta(T y) \leq d$, for all $y \in C$.

If $y \in C$, then for all $t \in[0, \infty)$ we have

$$
c \leq \psi(y) \leq y(t) \leq \frac{\psi(y)}{\ell} \leq \frac{\beta(y)}{\ell} \leq \frac{d}{\ell} .
$$

Hence for all $t \in[0, \infty)$ we have $\ell^{2} d=c \leq y(t) \leq \frac{d}{\ell}$, so that by condition (b) we see that 


$$
\begin{aligned}
\beta(T y) & =\frac{1}{\omega} \int_{0}^{\omega} \int_{t}^{t+\omega} G(t, s) f(s, y(s)) d s d t \leq \frac{g^{*}}{\omega} \int_{0}^{\omega} \int_{t}^{t+\omega} f(s, y(s)) d s d t \\
& =\frac{g^{*}}{\omega} \int_{0}^{\omega} \int_{0}^{\omega} f(s, y(s)) d s d t=g^{*} \int_{0}^{\omega} f(s, y(s)) d s \\
& \leq g^{*} \int_{0}^{\omega}\left(m_{l}(\ell d)+b_{l}\right) d s=g^{*} \omega\left(m_{l}(\ell d)+b_{l}\right) \\
& \leq g^{*} \omega\left(m_{l} d+b_{l}\right)=\frac{1}{2} d\left[1+2 \ell-\ell^{2}\right] \leq d
\end{aligned}
$$

since $\ell \in(0,1)$.

Claim 4: $\beta(T y) \leq d$, for all $y \in A$ with $\psi(T y)<c$.

Let $y \in A$ with $\psi(T y)<c$. Then using Lemma 2.7 again, we have $T y(s) \leq c / \ell$ for all $s \in[0, \omega]$, hence

$$
\beta(T y)=\frac{1}{\omega} \int_{0}^{\omega} T y(s) d s \leq \frac{1}{\omega} \int_{0}^{\omega} \frac{c}{\ell} d s=\frac{c}{\ell}=\ell d<d .
$$

Therefore, the hypotheses of Theorem 3.2 have been satisfied; thus the operator $T$ has at least one positive solution $y^{*} \in A(\alpha, \beta, a, d)=A\left(\alpha, \beta, \ell^{2} d, d\right)$.

\section{Analogous Discrete Periodic Problem}

In this section we develop a parallel discrete discussion for the second-order periodic difference equation with variable periodic potentials given by

$$
\left\{\begin{array}{l}
-\Delta(p \Delta y)(t)-\left(\frac{r(t) p(t)}{1+r(t)}\right) \Delta y(t)+\left(\frac{q(t)}{1+r(t)}\right) y(t+1)=f(t, y(t+1)), \quad t \in \mathbb{Z} \\
y(t)=y(t+\omega), \quad \Delta y(t)=\Delta y(t+\omega), \quad t \in \mathbb{Z}
\end{array}\right.
$$

where $\Delta y(t)=y(t+1)-y(t)$, and $\omega \in \mathbb{N}$ is the integral period. Recent papers on the existence of solutions to second-order periodic difference equations include Atici and Cabada [1], Atici, Cabada, and Otero-Espinar [2], Atici and Guseinov [3], Guo, Yu, and Zuo [8], and Li and Zhang [12]; all of these papers assume $r(t) \equiv 0$.

Similar to Theorem 2.2, we have the following theorem for difference equations. The proof of this and subsequent results in this section are straightforward imitations of the proofs in previous sections.

Theorem 4.1. Assume that

$\left(H_{1}^{\prime}\right) p(t), q(t)>0$ and $r(t) \geq 0$ for all $t \in \mathbb{Z}$;

$\left(H_{2}^{\prime}\right) p(t)=p(t+\omega), q(t)=q(t+\omega), r(t)=r(t+\omega)$, for all $t \in \mathbb{Z}$. 
Define $[0, \omega]_{\mathbb{Z}}:=\{0,1,2, \cdots, \omega-1, \omega\}$, and

$$
\begin{aligned}
& p_{*}=\min _{t \in[0, \omega]_{\mathbb{Z}}} p(t), \quad q_{*}=\min _{t \in[0, \omega]_{\mathbb{Z}}} q(t), \quad r_{*}=\min _{t \in[0, \omega]_{\mathbb{Z}}} r(t), \\
& p^{*}=\max _{t \in[0, \omega]_{\mathbb{Z}}} p(t), \quad q^{*}=\max _{t \in[0, \omega]_{\mathbb{Z}}} q(t), \quad r^{*}=\max _{t \in[0, \omega]_{\mathbb{Z}}} r(t), \\
& \beta_{*}=\frac{q_{*}-p_{*} r^{*}+\sqrt{q_{*}^{2}+\left(p_{*} r^{*}\right)^{2}+2 p_{*} q_{*}\left(2+r^{*}\right)}}{2\left(1+r^{*}\right)}, \\
& \beta^{*}=\frac{q^{*}-p^{*} r_{*}+\sqrt{q^{* 2}+\left(p^{*} r_{*}\right)^{2}+2 p^{*} q^{*}\left(2+r_{*}\right)}}{2\left(1+r_{*}\right)} .
\end{aligned}
$$

If the potentials $p, q, r$ are such that $\beta_{*} \leq \beta^{*}$, then the $\omega$-periodic Riccati difference equation

$$
\Delta z(t)=q(t)-r(t) z(t+1)-\frac{z^{2}(t)}{p(t)+z(t)}, \quad t \in\{0,1,2, \cdots\},
$$

has an attracting positive $\omega$-periodic solution $\bar{z}$ such that $\beta_{*} \leq \bar{z}(t) \leq \beta^{*}$ for all $t \in\{0,1,2, \cdots\}$.

Remark 4.2. If the potentials $p, q, r$ are all constant on $\mathbb{Z}$ with $p \equiv 1$, then the Riccati equation (4.2) has a constant attracting solution given by

$$
\bar{z}=\beta_{*}=\beta^{*}=\frac{q-r+\sqrt{q^{2}+r^{2}+2 q(2+r)}}{2(1+r)} .
$$

Again, in parallel with Theorem 2.4 we have the following discrete theorem.

Theorem 4.3. Assume $\left(H_{1}^{\prime}\right),\left(H_{2}^{\prime}\right)$. Further assume that the potentials $p, q, r$ are such that $\beta_{*} \leq \beta^{*}$, where $\beta_{*}$ and $\beta^{*}$ are given in Theorem 4.1. Let $h: \mathbb{Z} \rightarrow \mathbb{R}$ be an $\omega$-periodic function, and let $z$ be the attracting positive $\omega$-periodic solution of (4.2). Then the periodic boundary value problem

$$
\Delta(p \Delta y)(t)+\left(\frac{r(t) p(t)}{1+r(t)}\right) \Delta y(t)-\left(\frac{q(t)}{1+r(t)}\right) y(t+1)+h(t)=0, \quad t \in\{0,1,2, \cdots\},
$$

with periodic boundary conditions

$$
y(t)=y(t+\omega), \quad \Delta y(t)=\Delta y(t+\omega), \quad t \in\{0,1,2, \cdots\},
$$

has periodic solution

$$
y(t)=\sum_{s=t}^{t+\omega-1} G(t, s) h(s)
$$

for the Green function $G(t, s)$ given by

$$
G(t, s):=\sum_{\tau=t}^{s} \frac{\prod_{j=t}^{\tau}\left[\frac{p(j)}{p(j)+z(j)}\right] \prod_{j=\tau}^{s}[\xi(j)+1]}{k p(\tau)}+\sum_{\tau=s+1}^{t+\omega-1} \frac{\prod_{j=t}^{\tau}\left[\frac{p(j)}{p(j)+z(j)}\right] \prod_{j=\tau}^{s+\omega}[\xi(j)+1]}{k p(\tau)},
$$

where $t \in\{0,1,2, \cdots\}, s \in\{t, t+1, \cdots, t+\omega-1\}$, the positive function $\xi$ is given by

$$
\xi(t):=\frac{z(t)}{p(t)}(1+r(t))+r(t)>0, \quad t \in\{0,1,2, \cdots\},
$$


and the constant $k$ is given by

$$
k:=\left(1-\prod_{j=0}^{\omega-1}\left[\frac{p(j)}{p(j)+z(j)}\right]\right)\left(\prod_{j=0}^{\omega-1}[\xi(j)+1]-1\right)>0 .
$$

In order to develop a discrete version of the results in [7], we have the following corollary.

Corollary 4.4. If $h: \mathbb{Z} \rightarrow \mathbb{R}$ is an $\omega$-periodic function, then the discrete periodic boundary value problem with dependence on a parameter $\rho$ given by

$$
\Delta^{2} y(t)-\rho^{2} y(t+1)+h(t)=0, \quad t \in \mathbb{Z},
$$

with periodic boundary conditions

$$
y(t)=y(t+\omega), \quad \Delta y(t)=\Delta y(t+\omega), \quad t \in \mathbb{Z},
$$

has periodic solution for $t \in \mathbb{Z}$ given by

$$
y(t)=\sum_{s=t}^{t+\omega-1} G(t, s) h(s), \quad G(t, s):=\frac{r^{2 t+\omega}+r^{2 s+2}}{r^{t+s}\left(r^{2}-1\right)\left(r^{\omega}-1\right)},
$$

where

$$
r:=\frac{1}{2}\left(2+\rho^{2}+\rho \sqrt{\rho^{2}+4}\right) .
$$

Lemma 4.5. Assume $\left(H_{1}^{\prime}\right)$ and $\left(H_{2}^{\prime}\right)$. Let $h: \mathbb{Z} \rightarrow[0, \infty)$ and let $z, y$ be the solutions of (4.2), (4.4) respectively. Then

$$
y(t) \geq \ell\|y\| \quad \text { for } \quad t \in[0, \omega]
$$

where the constant $\ell$ is given by

$$
\ell:=\frac{1}{\prod_{j=0}^{\omega-1}[\xi(j)+1]\left(1+\prod_{j=0}^{\omega-1}[\xi(j)+1]\right)}
$$


Proof. For the kernel $G$ given in (4.6), we have for $t \leq s \leq t+\omega-1$ that

$$
\begin{aligned}
G(t, s) & =\sum_{\tau=t}^{s} \frac{\prod_{j=t}^{\tau}\left[\frac{p(j)}{p(j)+z(j)}\right] \prod_{j=\tau}^{s}[\xi(j)+1]}{k p(\tau)}+\sum_{\tau=s+1}^{t+\omega-1} \frac{\prod_{j=t}^{\tau}\left[\frac{p(j)}{p(j)+z(j)}\right] \prod_{j=\tau}^{s+\omega}[\xi(j)+1]}{k p(\tau)} \\
& \leq \sum_{\tau=t}^{t+\omega-1} \frac{\prod_{j=t}^{\tau}\left[\frac{p(j)}{p(j)+z(j)}\right]}{k p(\tau)}\left(\prod_{j=\tau}^{s}[\xi(j)+1]+\prod_{j=\tau}^{s+\omega}[\xi(j)+1]\right) \\
& \leq\left(\prod_{j=t}^{s}[\xi(j)+1]+\prod_{j=t}^{s+\omega}[\xi(j)+1]\right) \sum_{\tau=t}^{t+\omega-1} \frac{\prod_{j=t}^{\tau}\left[\frac{p(j)}{p(j)+z(j)}\right]}{k p(\tau)} \\
& \leq \prod_{j=0}^{\omega-1}[\xi(j)+1]\left(1+\prod_{j=0}^{\omega-1}[\xi(j)+1]\right) \sum_{\tau=0}^{\omega-1} \frac{\prod_{j=0}^{\tau}\left[\frac{p(j)}{p(j)+z(j)}\right]}{k p(\tau)}
\end{aligned}
$$

by the periodicity of the functions involved. Likewise

$$
\begin{aligned}
G(t, s) & =\sum_{\tau=t}^{s} \frac{\prod_{j=t}^{\tau}\left[\frac{p(j)}{p(j)+z(j)}\right] \prod_{j=\tau}^{s}[\xi(j)+1]}{k p(\tau)}+\sum_{\tau=s+1}^{t+\omega-1} \frac{\prod_{j=t}^{\tau}\left[\frac{p(j)}{p(j)+z(j)}\right] \prod_{j=\tau}^{s+\omega}[\xi(j)+1]}{k p(\tau)} \\
& \geq \sum_{\tau=t}^{t+\omega-1} \frac{\prod_{j=t}^{\tau}\left[\frac{p(j)}{p(j)+z(j)}\right]}{k p(\tau)}=\sum_{\tau=0}^{\omega-1} \frac{\prod_{j=0}^{\tau}\left[\frac{p(j)}{p(j)+z(j)}\right]}{k p(\tau)} .
\end{aligned}
$$

If we define

$$
g_{*}:=\sum_{\tau=0}^{\omega-1} \frac{\prod_{j=0}^{\tau}\left[\frac{p(j)}{p(j)+z(j)}\right]}{k p(\tau)}
$$

and

$$
g^{*}:=\prod_{j=0}^{\omega-1}[\xi(j)+1]\left(1+\prod_{j=0}^{\omega-1}[\xi(j)+1]\right) \sum_{\tau=0}^{\omega-1} \frac{\prod_{j=0}^{\tau}\left[\frac{p(j)}{p(j)+z(j)}\right]}{k p(\tau)}
$$

then

$$
y(t) \geq \frac{g_{*}}{g^{*}}\|y\|=\ell\|y\|, \quad t \in\{0,1,2, \cdots\} .
$$

This completes the proof.

An existence result for the discrete periodic boundary value problem (4.1) in the spirit of Theorem 3.3 is now possible. 


\section{REFERENCES}

[1] F.M. Atici and A. Cabada, Existence and uniqueness results for discrete second-order periodic boundary value problems, Computers Math. Appl., 45(6-9) (2003) 1417-1427.

[2] F.M. Atici, A. Cabada, and V. Otero-Espinar, Criteria for existence and nonexistence of positive solutions to a discrete periodic boundary value problem, J. Difference Equ. Appl., 9 (2003) 765-775.

[3] F.M. Atici and G.Sh. Guseinov, Positive periodic solutions for nonlinear difference equations with periodic coefficients, J. Math. Anal. Appl., 232 (1999) 166-182.

[4] D.R. Anderson, Multiple periodic solutions for a second-order problem on periodic time scales, Nonlinear Anal., 60:1 (2005) $101-115$.

[5] R. I. Avery, J. Henderson and D. R. Anderson, A topological proof and extension of the Leggett-Williams fixed point theorem, Comm. Appl. Nonlinear Anal., 16, No. 4 (2009), 39-44.

[6] J.F. Chu, P.J. Torres, and M.R. Zhang, Periodic solutions of second order non-autonomous singular dynamical systems $J$. Differential Equations, 239 (2007) 196-212.

[7] J.R. Graef, L. Kong, and H. Wang, Existence, multiplicity, and dependence on a parameter for a periodic boundary value problem, J. Differential Equations, 245 (2008) 1185-1197.

[8] Z.M. Guo, J.S. Yu, and X. Zuo, Periodic solutions of second order self-adjoint difference equations, J. London Math. Soc., 71 (2005) 146-160.

[9] W. Kelley and A. Peterson, The Theory of Differential Equations: Classical and Qualitative, Pearson Prentice Hall, Upper Saddle River, NJ, 2004.

[10] M.A. Krasnosel'skii, Positive Solutions of Operator Equations, P. Noordhoff Ltd., Groningen, The Netherlands, 1964.

[11] R.W. Leggett and L.R. Williams, Multiple positive fixed points of nonlinear operators on ordered Banach spaces, Indiana University Math. J., 28 (1979) 673-688.

[12] X. Li and J. Zhang, Periodic solutions of some second order difference equations, J. Difference Equations Appl., 15(6) (2009) $579-593$.

[13] Y. Liu, W. Ge, and Z. Gui, Three positive periodic solutions of nonlinear differential equations with periodic coefficients, Anal. Appl., 3(2) (2005) 145-155.

[14] P. Torres, Existence of one-signed periodic solutions of some second-order differential equations via a Krasnoselskii fixed point theorem, J. Differential Equations, 190 (2003) 643-662.

Department of Mathematics and Computer Science, Concordia College, Moorhead, Mn 56562 USA

E-mail address: andersod@cord.edu

URL: http://www.cord.edu/faculty/andersod/

College of Arts and Sciences, Dakota State University, Madison, South Dakota 57042 USA

E-mail address: rich.avery@dsu.edu

$U R L:$ http://www.homepages.dsu.edu/averyr/ 